\title{
Efficacy and Safety of an Extended Nevirapine Regimen in Infants of Breastfeeding Mothers With HIV-1 Infection for Prevention of HIV-1 Transmission (HPTN 046): 18-Month Results of a Randomized, Double-Blind, Placebo-Controlled Trial
}

\author{
Mary Glenn Fowler, MD, MPH, * Hoosen Coovadia, MD, $\dagger$ Casey M. Herron, MS, $\neq$ \\ Yvonne Maldonado, MD, $\S$ Tsungai Chipato, MBChB,\| Dhayendre Moodley, PhD, 1 \\ Philippa Musoke, MBchB, PhD,\# Jim Aizire, MBChB, MS,\# Karim Manji, MBBS,** \\ Lynda Stranix-Chibanda, MBChB, MMed, $+\dagger$ Wafaie Fawzi, MD, + Vani Chetty, B Tech, 9 \\ Lindiwe Msweli, BA, $\mid$ Rodrick Kisenge, PhD,** Elizabeth Brown, ScD, $\neq$ Anthony Mwatha, MS, $\neq$ \\ Susan H. Eshleman, MD, * Paul Richardson, MSc, * Melissa Allen, MPH,\# Kathleen George, MPH,ft \\ Philip Andrew, RN, $\S \S$ Sheryl Zwerski, CRNP, ||| Lynne M. Mofenson, MD, 99 \\ and J. Brooks Jackson, MD, MBA,* for the HPTN 046 Protocol Team
}

\begin{abstract}
Background: HPTN 046 compared the efficacy and safety of infant nevirapine (NVP) among HIV-exposed breastfed infants randomized at 6 weeks to 6 months to t NVP or placebo to prevent postnatal infection: we report final 18 -month outcomes.
\end{abstract}

Methods: Randomized, placebo-controlled trial in 4 African countries. Infant diagnostic HIV testing was performed regularly from birth through 18 months. Kaplan-Meier analysis was used to assess 18-month cumulative infant HIV infection, HIV infection/or death, and mortality rates.

Results: Between 6 weeks and 6 months, postnatal HIV infection rates were significantly lower among infants receiving daily NVP from 6 weeks to 6 months $1.1 \%$ [ $95 \%$ confidence interval (CI): $0.2 \%$ to $1.8 \%$ ], compared with placebo $2.4 \%$ (95\% CI: $1.3 \%$ to $2.6 \%), P=0.049$, but not significantly lower thereafter. Eighteen-month postnatal infection rates were low: $2.2 \%$ (95\% CI: $1.1 \%$ to $3.3 \%)$ versus $3.1 \%(95 \% \mathrm{CI}$ : $1.9 \%$ to $4.4 \%$ ), respectively, $P=0.28$. Mortality and HIV infection/death did not differ between arms at any age. Infants of women receiving antiretroviral therapy (ART) for their own health had the lowest 18month postnatal infection rates $(0.5 \%, 95 \%$ CI: $0.0 \%$ to $1.1 \%)$. However, HIV infection/death rates at 18 months were not significantly different for infants of mothers on ART $(3.7 \%, 95 \%$ CI: $1.9 \%$ to $5.5 \%$ ), and infants of mothers with CD4 counts of $\geq 350$ cells per cubic millimeter not receiving ART $(4.8 \%, 95 \% \mathrm{CI}$ : $2.7 \%$ to $6.8 \% ; P=0.46)$. There were no differences in adverse events between study arms.

Received for publication October 25, 2013; accepted October 25, 2013.

From the *Department of Pathology, Johns Hopkins University School of Medicine, Baltimore, MD; †Maternal Adolescent and Child Health, University of the Witwatersrand, South Africa; †Fred Hutchinson Cancer Research Center, Seattle, WA; §Department of Pediatrics, Stanford University School of Medicine, Stanford, CA; |Department of Obstetrics and Gynecology, College of Health Sciences, University of Zimbabwe, Harare, Zimbabwe; 9 Centre for the AIDS Programme of Research in South Africa (CAPRISA), Nelson R Mandela School of Medicine, University of KwaZulu Natal, Durban, South Africa; \#Makerere University, Johns Hopkins University, Research Collaboration, Kampala, Uganda; **Muhimbili University of Health and Allied Sciences, Dar es Salaam, Tanzania; $\uparrow \dagger$ Department of Pediatrics, College of Health Sciences, University of Zimbabwe, Harare, Zimbabwe; + †Department of Global Health and Population, Harvard University School of Public Health, Boston, MA; § \$Family Health International, Research Triangle Park, NC; |||Division of HIV/AIDS, National Institute of Allergy and Infectious Diseases, US National Institutes of Health, Bethesda, MD; and q $\uparrow$ Maternal and Pediatric Infectious Disease Branch, Eunice Kennedy Shriver National Institute of Child Health and Human Development, National Institutes of Health, Rockville, MD.

Supported by the National Institutes of Health (National Institute of Allergy and Infectious Diseases, and Eunice Kennedy Shriver National Institute of Child Health and Human Development) Grant U01 AI068632. HIV Prevention Trials Network (HPTN) 046 (ClinicalTrials.gov Identifier: NCT00074412) was funded by the US National Institutes of Health, initially through the HPTN and later through the International Maternal Pediatric Adolescent AIDS Clinical Trials (IMPAACT) group. The HPTN (U01AI46749) has been funded by the National Institute of Allergy and Infectious Diseases (NIAID), the Eunice Kennedy Shriver National Institute of Child Health and Human Development (NICHD), National Institute of Drug Abuse, and National Institute of Mental Health (NIMH). The IMPAACT Group (U01AI068632) has been funded by NIAID, NICHD, and NIMH. The study products were provided free of charge by Boehringer Ingelheim Pharmaceuticals.

Presented at the 20th Conference on Retroviruses and Opportunistic Infections (CROI 2013), March 5, 2013, Atlanta, GA. Abstract no. U-1003.

The authors have no conflicts of interest to disclose.

The conclusions and opinions expressed in this article are those of the authors and do not necessarily reflect those of the National Institutes of Health or U.S. Department of Health and Human Services.

Correspondence to: Mary Glenn Fowler, MD, MPH, Department of Pathology, Johns Hopkins University School of Medicine, Baltimore, MD 21205 (e-mail: mgfowler@mujhu.org).

Copyright (C) 2013 by Lippincott Williams \& Wilkins

366 | www.jaids.com

J Acquir Immune Defic Syndr • Volume 65, Number 3, March 1, 2014 
Conclusions: This trial demonstrated early but not late differences in postnatal HIV transmission among infants randomized at age 6 weeks to extended NVP or placebo, underscoring the importance of continued prophylaxis throughout breastfeeding.

Key Words: nevirapine, infant HIV prophylaxis, PMTCT

(J Acquir Immune Defic Syndr 2014;65:366-374)

\section{INTRODUCTION}

In high HIV-prevalence resource-limited settings, breastfeeding is critical to child survival but also carries the risk of HIV transmission for HIV-exposed infants. In 2011, UNAIDS estimated that 330,000 infants were infected with $\mathrm{HIV}$, one-third of these occurring during breastfeeding. ${ }^{1}$ The global health goal is to find strategies that optimize breastfeeding benefits while reducing the risk of HIV transmission during breastfeeding.

Toward this goal, several recent randomized clinical trials conducted among HIV-infected breastfeeding women demonstrated the efficacy of either maternal triple antiretroviral (ARV) drug prophylaxis or infant ARV prophylaxis for up to 28 weeks of breastfeeding. ${ }^{2-6}$

Revised World Health Organization (WHO) guidelines in 2009-2010 recommended exclusive breastfeeding through 6 months with extended breastfeeding through 12 months for HIV-exposed infants; and several ARV options for prevention of mother to child transmission (PMTCT) extrapolated from the above PMTCT prophylaxis trials. ${ }^{7,8}$ These options included option A: maternal zidovudine (AZT) starting as early as 14 weeks gestation followed by daily infant nevirapine (NVP) continuing until 1 week postcessation of breastfeeding; option $\mathrm{B}$ : maternal triple ARV prophylaxis during pregnancy and until 1 week postcessation of breastfeeding; or based on updated 2012-2013 WHO guidelines, option B+ with lifetime continuation of ART for all pregnant HIV-infected women. ${ }^{9,10}$

Among the infant based PMTCT prophylaxis trials, HPTN 046 was the only trial designed to test the relative efficacy and safety of extended once daily infant NVP prophylaxis to 6 months among breastfed HIV-exposed infants who had received 6 weeks of NVP and were uninfected at age 6 weeks. The trial was implemented during a time when breastfeeding was recommended to stop by 6 months for HIV-exposed infants.

Early results from HPTN 046 have been reported and demonstrated a significant reduction in postnatal transmission at age 6 months with the longer infant NVP regimen and no shortterm safety concerns. ${ }^{11}$ The aim of these analyses was to look at longer-term safety and late 18-month efficacy of the 6 months versus 6 weeks of infant NVP among HIV-exposed infants whose mothers were counseled to stop breastfeeding at 6 months. This article presents final HIV transmission, infant HIV-free survival, and overall infant survival through age 18 months, as well as cumulative adverse events (AEs) by study arm after discontinuation of infant study drug prophylaxis at 6 months.

\section{METHODS}

HPTN 046 was a phase 3, randomized, double-blind, placebo-controlled trial that assessed the efficacy and safety of extension of once-daily NVP to 6 months of age or until cessation of breastfeeding (whichever came first) for prevention of HIV transmission in HIV-1-exposed breastfeeding infants who had received NVP prophylaxis until 6 weeks of age. Eligible HIV-uninfected infants were then randomized to receive either extended daily NVP or placebo using age-adjusted doses through 6 months or end of breastfeeding, whichever occurred earlier. As per WHO and Ministries of Health recommendations at the time of the trial design, HIV-infected mothers were counseled to stop breastfeeding at about 6 months over a short time period. Both regimens exceeded country PMTCT guidelines in the sites at the time the study was implemented.

\section{Study Population}

HIV-infected mothers were recruited at trial sites in South Africa, Tanzania, Uganda, and Zimbabwe. Interested HIV-infected women aged $\geq 18$ years without other serious illness who intended to breastfeed were consented and screened either during the third trimester or up through 7 days postpartum. There were no restrictions to entry based on maternal CD4 counts, and women could have received either ARVs solely for PMTCT or for treatment. Written informed consent was obtained from HIV-infected eligible mothers. Eligible women and their infants were enrolled within 7 days of delivery. Infants were eligible if HIV-uninfected based on an HIV DNA polymerase chain reaction (PCR) negative from specimen obtained within 7 days of birth, birth weight of $\geq 2000 \mathrm{~g}$, without life-threatening conditions and breastfeeding. Infant follow-up study visits were scheduled at 2, 5, 6, and 8 weeks, and at 3, 6, 9, 12, and 18 months. Enrolled women were counseled to exclusively breastfeed for 6 months, and then wean rapidly with introduction of breast milk substitutes and complementary foods. A detailed description of the HPTN 046 study design has been previously reported. ${ }^{11}$ The trial registration number is NCT00074412 under clinicaltrials.gov registry, and the implemented study protocol can be accessed at www.HPTN.org.

Study recruitment into version 3.0 of HPTN 046 began in May 2008 after protocol approval by all relevant Institutional Review Boards and regulatory bodies in the respective countries. Eighteen-month infant follow-up was completed in September 2011.

\section{Randomization and Masking}

All enrolled infants received once-daily open-label NVP $(10 \mathrm{mg} / \mathrm{mL}$ oral suspension) for the first 6 weeks of life. After the initial 6-week NVP prophylaxis regimen, at between 6 and 8 weeks, eligible infants were randomized in a one-to-one ratio to receive extended NVP (treatment arm) or placebo (control arm) according to computer-generated permutated block algorithms by site with random block sizes. Infants were also stratified by maternal ART use at randomization. Study staff and participants were masked to the study product (NVP or placebo) assignment.

\section{Procedures}

For infants randomized at 6 weeks, real-time PCR HIV-1 testing was performed at 3, 6, 9, and 12 months, and 
EIA or rapid HIV antibody test at 18 months. An infant was considered HIV-1 infected at months 3 through 12, if 2 separate blood specimens taken at different times were positive by HIV-1 DNA PCR (Roche AMPLICOR HIV-1 DNA Test, Version 1.5) or quantitative HIV-1 RNA PCR, if HIV-1 DNA PCR was not available. An infant was considered HIV-1-infected at month 18, if 2 separate blood specimens taken at different times were positive by HIV EIA or rapid testing and confirmed by HIV-1 Western blot (Bio-Rad Laboratories Genetic Systems HIV-1 Western Blot). Infant stored specimens collected at randomization were tested retrospectively if the infant had a positive PCR test at the 3-month visit; those infants found to have a positive test at randomization were excluded from the analyses. All infants received cotrimoxazole as standard of care according to their country guidelines.
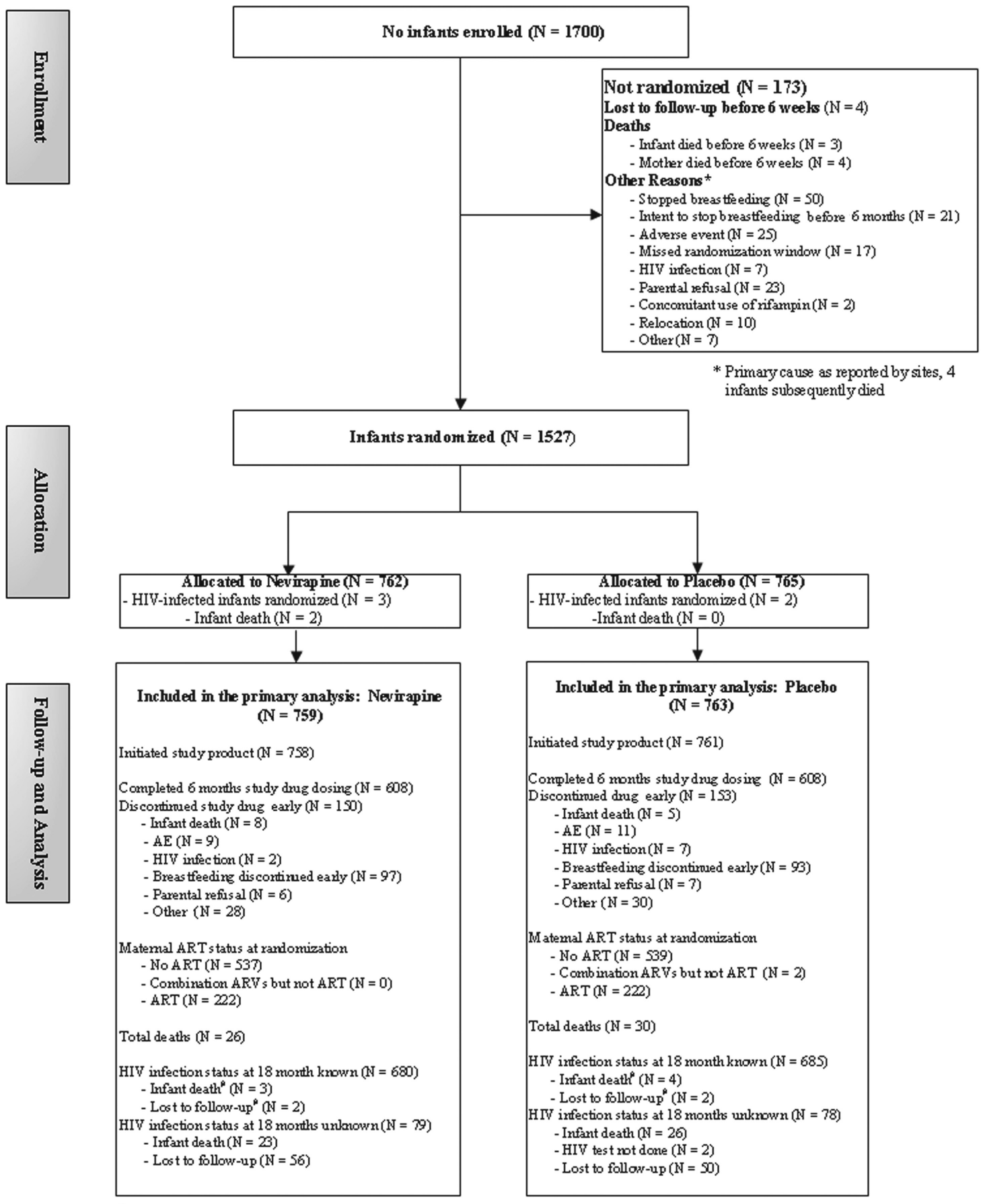

${ }^{*} \mathrm{HIV}$ infection was confirmed before to death or loss to follow-up

FIGURE 1. Study design and participant follow-up. 
All AEs were captured through 8 months of infant life ( 8 weeks after the maximum study drug dosing duration) and thereafter through 18 months, only if considered serious or meeting criteria for expedited reporting. We graded clinical and laboratory AEs according to the DAIDS Table for grading the severity of adult and pediatric AEs (Version 1.0, December 2004, with Clarification dated August 2009). ${ }^{12}$

Final primary study end points included cumulative infant HIV-1 infection at age 18 months among infants who were uninfected at age 6 weeks and relative rates of HIV-1 infection over 18 months, HIV-free and overall survival at 18 months, and cumulative frequency and severity of AEs by 18 months. Adherence to study product was based on maternal self-report.

\section{Statistical Analysis}

Sample size determination and statistical methods are described in detail elsewhere. ${ }^{11}$ Means and proportions were used to summarize baseline characteristics. The Kaplan-Meier method was used to calculate rates of cumulative HIV-1 infection, death, and HIV-free survival by randomization arm at 6 , 9,12 , and 18 months among infants uninfected at 6 weeks of age, and Greenwood formula was used to calculate standard errors at the defined time points. We used a $\mathrm{Z}$ statistic to compare the calculated rates at each time point. For the Kaplan-Meier cumulative-infection analysis, time to HIV-1 infection was defined as the midpoint between the last negative and first positive test. We censored infants with no positive tests at the time of the last negative test. For HIV-free survival, the event time was the minimum of the infection time and the death time with censoring at the last negative test if no positive test or death was observed.
To better understand the impact of extended infant NVP prophylaxis on transmission risk, in the presence or absence of maternal ART; we also conducted post hoc exploratory nonrandomized subgroup analyses. For these subgroup analyses, we assessed subgroups defined by maternal ART use and maternal CD4 counts $\left(<350 / \geq 350\right.$ cells $\left./ \mathrm{mm}^{3}\right)$ at the time of randomization. Using the same statistical methods, an exploratory analysis was also performed to compare rates of death and/or HIV-free survival between infants born to mothers who were on ART at randomization, and infants who were randomized to the NVP arm whose mothers were not on ART and had CD4 counts of $\geq 350$ cells per cubic millimeter at randomization.

\section{Role of the Funding Source}

The sponsor (US National Institutes of Health) participated in the design and oversight of the study, interpretation of the results, and preparation of the article. The sponsor also provided for an independent data and safety monitoring board to review the trial safety and efficacy at regular intervals. Members of the writing team had full access to the study data. The protocol chair, co-chairs, and sponsor representatives had final responsibility for submission for publication.

\section{RESULTS}

Between June 2008 and March 2010, 1527 infants were randomized at age 6 weeks (following 6 week open-label daily NVP). Five infants ( 3 in NVP and 2 in placebo arm) who were later found to have a positive HIV-1 DNA PCR tested retrospectively on stored blood from age 6 weeks were excluded from the analysis. This left 1522 infants included in the primary analyses: 759 infants for the primary analyses in

TABLE 1. Cumulative 18-Month HIV-1 Infection, Death, or Both in Infants Uninfected at Age 6 Weeks

\begin{tabular}{|c|c|c|c|c|c|c|}
\hline \multirow{2}{*}{$\begin{array}{l}\text { Study End Point (at }>6 \\
\text { wk in Infants Uninfected } \\
\text { at } 6 \text { wk) }\end{array}$} & \multicolumn{2}{|c|}{ Extended NVP $(N=759)$} & \multicolumn{2}{|c|}{ Placebo $(N=763)$} & \multirow[b]{2}{*}{$\begin{array}{c}\text { Relative Risk } \\
\text { Reduction (\%) }\end{array}$} & \multirow[b]{2}{*}{$P \dagger$} \\
\hline & $\begin{array}{l}\text { Cumulative No. End } \\
\text { Points/No. at Risk }\end{array}$ & $\begin{array}{c}\text { Probability of End } \\
\text { Point }(\%)^{*}(95 \% \text { CI })\end{array}$ & $\begin{array}{l}\text { Cumulative No. End } \\
\text { Points/No. at Risk }\end{array}$ & $\begin{array}{c}\text { Probability of End } \\
\text { Point }(\%) *(95 \% \text { CI })\end{array}$ & & \\
\hline \multicolumn{7}{|l|}{ All randomized infants } \\
\hline \multicolumn{7}{|l|}{ HIV-1 infection } \\
\hline $6 \mathrm{mo}$ & $8 / 700$ & $1.1(0.3$ to 1.8$)$ & $18 / 698$ & $2.4(1.3$ to 3.6$)$ & 54 & 0.049 \\
\hline $9 \mathrm{mo}$ & $11 / 692$ & $1.5(0.6$ to 2.4$)$ & $21 / 683$ & $2.9(1.7$ to 4.1$)$ & 48 & 0.078 \\
\hline $12 \mathrm{mo}$ & $15 / 670$ & $2.1(1.0$ to 3.1$)$ & $22 / 667$ & $3.0(1.8$ to 4.2$)$ & 30 & 0.265 \\
\hline $18 \mathrm{mo}$ & $16 / 664$ & $2.2(1.1$ to 3.3$)$ & $23 / 663$ & $3.1(1.9$ to 4.4$)$ & 29 & 0.280 \\
\hline \multicolumn{7}{|l|}{ Death } \\
\hline $6 \mathrm{mo}$ & $9 / 716$ & $1.2(0.4$ to 2.0$)$ & $8 / 725$ & $1.1(0.3$ to 1.8$)$ & 0 & 0.805 \\
\hline $9 \mathrm{mo}$ & $16 / 703$ & $2.2(1.1$ to 3.2$)$ & $19 / 705$ & $2.6(1.4$ to 3.7$)$ & 15 & 0.612 \\
\hline $12 \mathrm{mo}$ & $20 / 688$ & $2.7(1.6$ to 4.0$)$ & $26 / 690$ & $3.5(2.2$ to 4.9$)$ & 23 & 0.373 \\
\hline $18 \mathrm{mo}$ & $26 / 678$ & $4.7(2.0$ to 7.4$)$ & $30 / 684$ & $4.1(2.7$ to 5.6$)$ & -15 & 0.719 \\
\hline \multicolumn{7}{|l|}{ HIV-1infection or death } \\
\hline $6 \mathrm{mo}$ & $17 / 706$ & $2.3(1.2$ to 3.4$)$ & $24 / 707$ & $3.2(2.0$ to 4.5$)$ & 28 & 0.274 \\
\hline $9 \mathrm{mo}$ & $26 / 693$ & $3.6(2.2$ to 4.9$)$ & $39 / 687$ & $5.3(3.7$ to 6.9$)$ & 32 & 0.104 \\
\hline $12 \mathrm{mo}$ & $32 / 676$ & $4.5(3.0$ to 6.0$)$ & $46 / 669$ & $6.3(4.6$ to 8.1$)$ & 29 & 0.111 \\
\hline $18 \mathrm{mo}$ & $39 / 668$ & $5.5(3.8$ to 7.1$)$ & $49 / 665$ & 6.7 (4.9 to 8.5$)$ & 18 & 0.323 \\
\hline
\end{tabular}

*Cumulative rates at $6,9,12$, and 18 months calculated using Kaplan-Meier methods.

$\dagger$ Two-sided $P$ value for $\mathrm{Z}$ statistic of the difference in probabilities. 

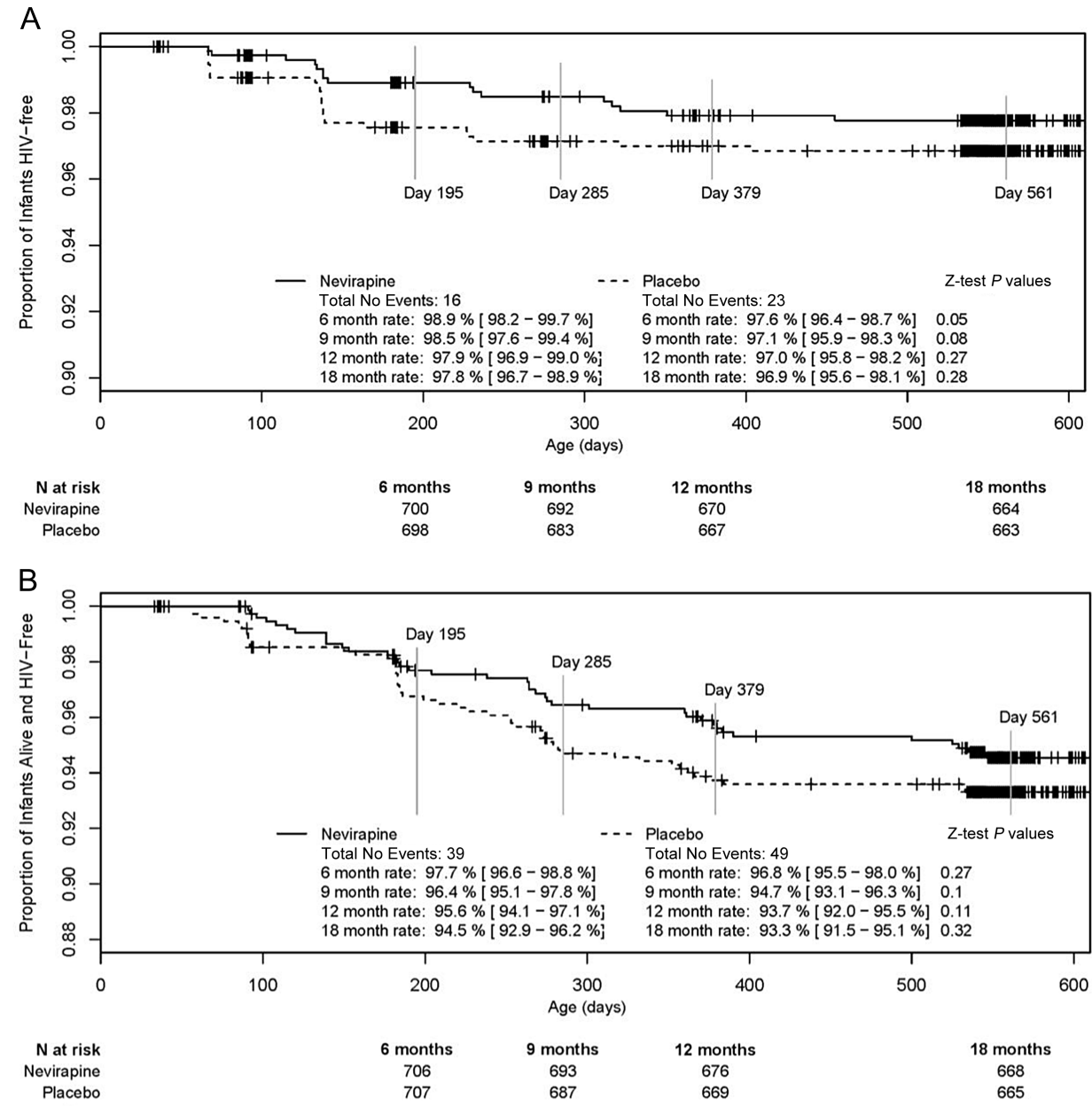

C

FIGURE 2. A, Proportion of infants HIV-free. B, Proportion of infants alive and HIV-free. C, Proportion of infants alive.

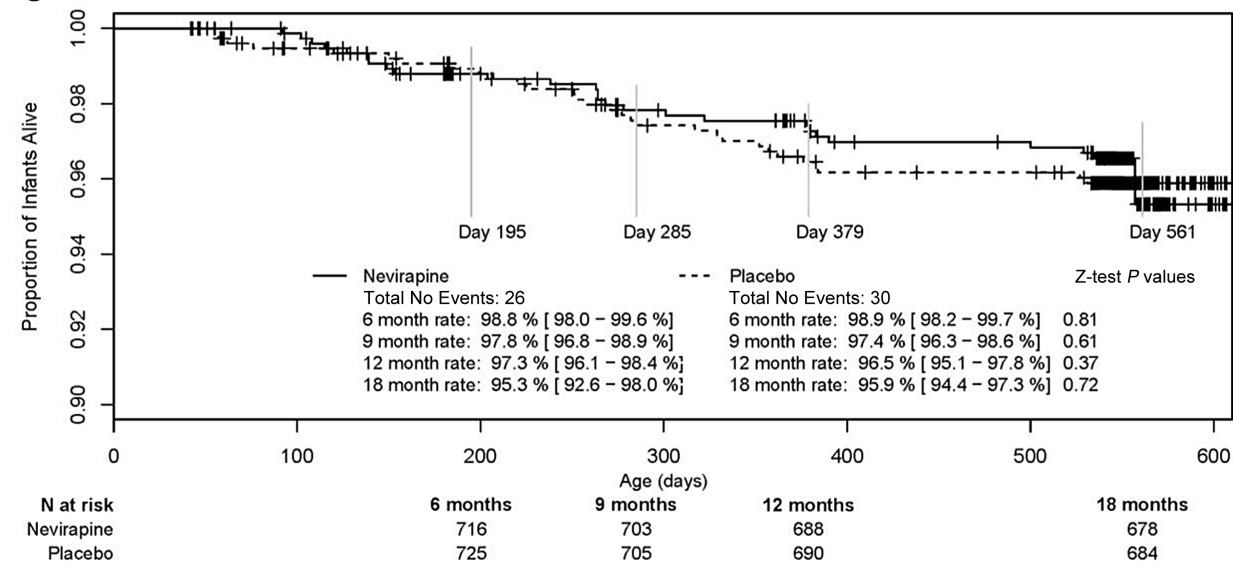

the NVP arm and 763 infants in the placebo arm (Fig. 1). Maternal and infant demographics and maternal clinical status were similar across the 2 study arms at randomization: median maternal age was 27 years and median infant birth weight was $3100 \mathrm{~g}$ in both study product arms; $96 \%$ and $97 \%$ of women were WHO clinical stage I or II and median CD4 count was 560 (range, 53-2172) and 528 (range, 49-1962) cells per cubic millimeter in the NVP and placebo arms, respectively. Four hundred thirty-nine $(29 \%)$ mothers were receiving ART for their own health at the 6-week randomization visit, 220/752 $(29 \%)$ on the NVP arm and 219/753 (29\%) on the placebo arm. Fifty-two percent of infants in each study arm were men. ${ }^{11}$

During the 6-week to 18-month follow-up period among randomized infants, there were 56 infant deaths, 26 in the NVP and 30 in the placebo arms. Excluding deaths, overall loss to follow-up at 18 months was low across both 
TABLE 2. AEs, Selected Grade 3 or Higher Laboratory Abnormalities, and Rash AEs Overall and by Study Arm, Among Randomized Infants Who Were Exposed to Study Product (NVP or Placebo)

\begin{tabular}{|c|c|c|c|c|}
\hline & Nevirapine & Placebo & Total & $\boldsymbol{P}$ \\
\hline No. infants who initiated study product & 758 & 761 & 1519 & - \\
\hline \multicolumn{5}{|l|}{ AEs } \\
\hline Infants with at least $1 \mathrm{AE}$ & $629(83.0 \%)$ & $633(83.1 \%)$ & $1262(83.1 \%)$ & 0.918 \\
\hline Infants with at least $1 \mathrm{SAE}$ & $130(17.2 \%)$ & $126(16.6 \%)$ & $256(16.7 \%)$ & 0.758 \\
\hline Infants with at least $1 \mathrm{SAE}$ judged severe & $52(6.9 \%)$ & $52(6.8 \%)$ & $104(6.8 \%)$ & 0.983 \\
\hline Infants with at least $1 \mathrm{SAE}$ judged life threatening & $12(1.6 \%)$ & $20(2.6 \%)$ & $32(2.1 \%)$ & 0.156 \\
\hline Infants with at least $1 \mathrm{SAE}$ leading to death & $21(2.8 \%)$ & $21(2.8 \%)$ & $42(2.8 \%)$ & 0.990 \\
\hline Infants with an SAE judged possibly related to study drug & $2(0.3 \%)$ & $2(0.3 \%)$ & $4(0.3 \%)$ & 1.000 \\
\hline Death & $1(0.1 \%)$ & $1(0.1 \%)$ & $2(0.1 \%)$ & - \\
\hline Alanine aminotransferase increased & $0(0 \%)$ & $1(0.1 \%)$ & $1(0.1 \%)$ & - \\
\hline Neutrophil count decreased & $1(0.1 \%)$ & $0(0 \%)$ & $1(0.1 \%)$ & - \\
\hline Infants with an SAE judged probably related to study drug & $0(0 \%)$ & $0(0 \%)$ & $0(0 \%)$ & 1.000 \\
\hline Infants with grade $2 \mathrm{~B}$ or worse rash & $1(0.1 \%)$ & $0(0 \%)$ & $1(0.1 \%)$ & 0.499 \\
\hline No. infants with at least 1 safety laboratory assessment & 757 & 757 & 1514 & - \\
\hline \multicolumn{5}{|l|}{ Abnormal laboratory values* } \\
\hline Infants with any grade neutropenia & $458(60.5 \%)$ & $442(58.4 \%)$ & $900(59.4 \%)$ & 0.402 \\
\hline Infants with grade 3 or higher neutropenia & $161(21.3 \%)$ & $133(17.7 \%)$ & $294(19.4 \%)$ & 0.069 \\
\hline Infants with any grade anemia & $701(92.6 \%)$ & $674(89.0 \%)$ & $1375(90.8 \%)$ & 0.016 \\
\hline Infants with grade 3 or higher anemia & $188(24.8 \%)$ & $167(22.1 \%)$ & $355(23.4 \%)$ & 0.203 \\
\hline Infants with any grade ALT concentration & $28(3.7 \%)$ & $25(3.3 \%)$ & $53(3.5 \%)$ & 0.678 \\
\hline Infants with grade 3 or higher ALT concentration & $4(0.5 \%)$ & $3(0.4 \%)$ & $7(0.5 \%)$ & 1.000 \\
\hline
\end{tabular}

SAE, serious adverse event.

AEs (serious and nonserious) were collected through 8 months, whereas only SAEs were reported after 8 months through 18 months.

Based on 2004 DAIDS Toxicity Tables.

*Some laboratory values were associated with an AE.

study arms: $58 / 759(7.6 \%)$ in the NPV arm were lost to follow-up excluding deaths, and 52/763 (6.8\%) in the placebo arm. Maternal report of adherence to giving infant NVP was high. Across all visits, $88 \%-96 \%$ of mothers reported that their infants had missed no study product doses since the last visit, with no significant differences by study arm.

\section{Cumulative Transmission, HIV or Death, and Overall Mortality Through 18 Months}

Cumulative postnatal infant HIV infection, HIV infection/mortality rates, and overall mortality between age 6 weeks and 18 months are shown in Table 1 and presented as HIV-free, HIV-free survival, and overall 18-month survival rates in Figures $2 \mathrm{~A}-\mathrm{C}$. There were 39 postnatal HIV infections occurring between 6 weeks and 18 months. As reported earlier, HIV infection rates after age 6 weeks were low but significantly different at 6 months between study arms: $1.1 \%$ [95\% confidence interval (CI): $0.3 \%$ to $1.8 \%$ ] in the NVP arm versus $2.4 \%$ (95\% CI: $1.3 \%$ to $3.6 \%)$ in the placebo arm, $P=$ 0.049 . However, after discontinuation of study product at 6 months, HIV infection rates were no longer significantly different from 9 through 18 months. By 18 months, there were 16 infections in the NVP arm versus 23 infections in the placebo arm, with a cumulative postnatal infection rate of $2.2 \%$ (95\% CI: $1.1 \%$ to $3.3 \%$ ) versus $3.1 \%$ (95\% CI: $1.9 \%$ to $4.4 \%, P=0.28$ ), translating into HIV-free rates of $97.8 \%$ in the NVP arm versus $96.9 \%$ in the placebo arm (Fig. 2A).
Median duration of breastfeeding was 184 days in both study arms reflecting WHO guidelines at that time for HIVinfected mothers; by 12 months, $95 \%$ of infants had reported cessation of breastfeeding. A total of 14/39 (36\%) of infections occurred after age 6 months; $7 / 39(18 \%)$ infections $(5$ in the NVP arm and 2 in the placebo arm) were first detected between the ages 9 and 18 months, among infants with at least 1 negative PCR result recorded after reported cessation of breastfeeding.

Cumulative HIV-free survival rates between 6 weeks and 18 months are presented in Figure 2B; rates were not significantly different between study arms at $6,9,12$, or 18 months although there was a nonsignificant trend for higher risk of HIV infection and/or death among infants receiving placebo compared with NVP. The 18-month cumulative HIVfree survival rate was high: $94.5 \%$ (95\% CI: $92.9 \%$ to $96.2 \%)$ in the NVP arm and $93.3 \%$ (95\% CI: $91.5 \%$ to $95.1 \%)$ in the placebo arm $(P=0.32)$. Likewise, overall infant survival rates at 18 months (Fig. 2C) were not statistically different between study arms: $95.3 \%$ (95\% CI: $92.6 \%$ to $98.0 \%)$ for NVP versus 95.9\% (95\% CI: $94.4 \%$ to $97.3 \%)$ for placebo $(P=0.72)$.

\section{Post Hoc Subgroup Analyses: Rates of HIV Infection and HIV/Death by Maternal ART and CD4 Cell Subgroups}

In addition to the primary late outcomes, we calculated rates of infection for women by immune status and HIV treatment status to better understand the impact of infant NVP 
prophylaxis by maternal risk category. Analyses of infection rates among infants on each study arm and whose mothers were not on ART were stratified by maternal randomization CD4 cell counts of $<350$ cells per cubic millimeter versus those whose mothers with $\geq 350$ cells per cubic millimeter.

Among the 149 mothers (85 whose infants were randomized to the NVP arm and 64 to the placebo arm) with CD4 cell counts of $<350$ cells per cubic millimeter at randomization and not on ART, the cumulative infection rates were high, but not statistically different by study arm from 6 through 18 months: 18 months cumulative postnatal infection rates were $8.9 \%(95 \% \mathrm{CI}: 2.6 \%$ to $15.1 \%)$ and $9.6 \%(95 \% \mathrm{CI}$ : $2.3 \%$ to $17 \%)$ in the NVP and placebo arms, respectively $(P=$ 0.87). Likewise, there were no differences between study arms for this subgroup in 18 HIV infection and/or mortality.

For the 924 women with CD4 cell counts of $\geq 350$ cells per cubic millimeter at randomization and not considered eligible for ART (451 whose infants were randomized to the NVP arm and 473 whose infants were randomized to the placebo arm), cumulative postnatal infection risk was consistently lower in the infant NVP arm than the placebo arm from 6 through 18 months, although this trend was not statistically significant. Postnatal transmission risk at 18 months was $1.9 \%$ (95\% CI: $0.6 \%$ to $3.2 \%)$ in the NVP arm and $3.5 \%$ (95\% CI: $1.8 \%$ to $5.2 \%)$ in the placebo arm $(P=$ $0.14)$. Likewise, the overall 18 -month risk of HIV infection and/or death was lower in the NVP arm, 4.8\% (95\% CI: $2.7 \%$ to $6.8 \%$ ), compared with $7.2 \%$ (95\% CI: $4.9 \%$ to $9.6 \%, P=$ 0.120 ) in the placebo arm.

Among infants whose mothers were receiving ongoing ART according to their country guidelines, cumulative postnatal transmission rates between 6 weeks and 18 months were uniformly low $(0.5 \%$; $95 \%$ CI: $0.0 \%$ to $1.4 \%)$ irrespective of study arm. The 18-month risk of HIV infection and/or death was similar in both study arms, $3.7 \%$ (95\% CI: $1.2 \%$ to $6.2 \%$ ) in the NVP arm versus $3.8 \%$ (95\% CI: $1.2 \%$ to $6.3 \%$ ) in the placebo arm.

We also explored, if there were any differences at 6 through 18 months in HIV transmission and/or death between infants randomized to the NVP arm born to women with CD4 counts of $\geq 350$ cells per cubic millimeter not receiving ART and those infants whose mothers were on ART irrespective of infant study arm. We found no significant differences in HIVfree survival comparing these subgroups: the 18-month risk of HIV infection and/or death was $3.7 \%$ (95\% CI: $1.9 \%$ to $5.5 \%$ ) versus $4.8 \%$ (95\% CI: $2.7 \%$ to $6.8 \% ; P=0.460)$.

\section{Cumulative AEs and Serious Adverse Events}

Given high background rates of infectious diseases and malnutrition at the study sites where the HPTN 046 study was conducted, clinical AEs were common with $83 \%$ of infants in either arm reporting at least $1 \mathrm{AE}$ by 18 months (Table 2). Most clinical AEs were mild to moderate in nature; and the majority of all AEs ( $94 \%$ in each arm) were judged not related or probably not related to study product. There were no significant differences in infant AEs by study arm.

The most common laboratory abnormalities among infants were decreased hemoglobin $(\mathrm{Hb})$ and neutrophil counts occurring at least once in $91 \%$ and $59 \%$ of infants, respectively, with infants on NVP having a significantly increased risk of lower $\mathrm{Hb}$ (grade 1 or higher, $P=0.016$ ). Twenty-three percent of infants had at least 1 grade 3 or higher episode of low $\mathrm{Hb}$, and $19 \%$ infants had at least 1 episode of grade 3 or higher neutropenia. In general, these hematologic laboratory abnormalities were not associated with symptoms and resolved without intervention. Liver enzyme alanine aminotransferase (ALT) elevations were uncommon; $3.7 \%$ of infants receiving NVP and 3.3\% receiving placebo had grade 1 or higher ALT elevation. Grade 3 or 4 ALT elevations were rare; $0.5 \%$ (NVP) and $0.4 \%$ (placebo). Study product discontinuation was also rare and did not differ between arms (3\% in each arm); the most common reason for discontinuation was neutropenia.

\section{Infant Deaths}

There were 56 infant deaths from age 6 weeks to 18 months, representing an overall infant mortality of $4.3 \%$, with no statistical differences by study arm (26 deaths-NVP vs. 30 deaths - placebo), with cumulative mortality rates of $4.7 \%$ versus $4.1 \%$, respectively $(P=0.72)$. The most common causes of infant mortality were gastroenteritis, pneumonia, unknown cause, injuries, and anemia.

\section{DISCUSSION}

This article presents the final 18-month efficacy and safety results of HPTN 046, which compared extended infant daily NVP study product to placebo study product from age 6 weeks to 6 months among HIV-exposed breastfed infants all of whom received open-label NVP from birth to 6 weeks of age. As reported earlier, based on HIV transmissions between 6 weeks and 6 months of age, there was a statistically significant reduction in transmission risk for infants in the extended NVP arm (1.1\%) compared with those in the placebo arm (2.4\%) at the 6-month time point. ${ }^{11}$ However, there were no statistical differences in postnatal transmission rates between study arms for the remainder of the follow-up period from age 6 months through 18 months, after the infant prophylaxis had stopped. These results speak to the effectiveness of infant NVP prophylaxis during the time it was given during 6 months of breastfeeding but also show this effect was diluted over time after the infant ARV prophylaxis intervention was stopped.

The HPTN 046 maternal population was reflective of pregnant women seen in PMTCT programs where the women were recruited in South Africa, Tanzania, Uganda, and Zimbabwe. Most were young and had higher CD4 counts with a median CD4 at baseline of 560 cells per cubic millimeter. Twenty-nine percent of mothers was on ART according to their country guidelines at the time of study randomization. Subgroup analyses indicated very low postnatal transmission rates $(0.5 \%)$ for women who were on ART for their own care throughout the 18 months follow-up.

Also as seen in other studies of extended ARV prophylaxis used to reduce postnatal transmission, ${ }^{13,14}$ in HPTN 046 there were late unexplained transmissions, which 
were detected after reported breastfeeding cessation: 7 of 39 late transmissions were detected subsequent to at least 1 documented negative infant PCR result, and after maternal reported cessation of breastfeeding. This may have been due to continued unreported breastfeeding or could be due to as yet unexplained HIV exposures after breastfeeding cessation.

The cumulative 18-month follow-up rates of postnatal transmission between 6 weeks and 18 months were encouragingly low $(2.2 \%$ in the NVP arm and $3.1 \%$ in the placebo arm) in this breastfeeding HIV-exposed population, where all study infants received at least 6 weeks of daily NVP after birth and were counseled to stop breastfeeding at about 6 months. This postnatal transmission was substantially lower than reported historical studies in breastfeeding infants before ARV prophylaxis, where the cumulative overall risk of late postnatal infection was 8.9 transmissions per 100 child-years of breastfeeding, with $9.3 \%$ cumulative probability of infection between 4 weeks and 18 months. ${ }^{15}$

The HPTN 046 study results add to the body of literature, which supports the effectiveness of extended infant prophylaxis in reducing the risk of HIV transmission through breast milk. Although updated guidelines now recommend 12 months of breastfeeding for HIV-exposed infants, the HPTN 046 findings remain relevant in demonstrating the efficacy of infant NVP prophylaxis for the time it was given during breastfeeding.

Both extended infant and maternal prophylaxis have been shown to be effective in reducing postnatal transmission among women who do not require ART for their own care. ${ }^{3,4,11}$ Each approach has advantages and disadvantages.

Maternal triple ARV prophylaxis (eg, option B, option $\mathrm{B}+$ ) among women with higher CD4 cell counts decreases transmission to known noninfected discordant partners based on recent adult clinical trial data, ${ }^{16}$ and harmonizes with adult ART guidelines. Challenges of option B and option $\mathrm{B}+$ include risks of long-term complications with early initiation of ARVs among asymptomatic women and well-documented high loss to follow-up and decreased ARV adherence among HIV-infected pregnant and postpartum women in programmatic settings. ${ }^{17-20}$ Poor adherence and loss to follow-up in turn raise the risk of early emergence of multiclass ARV resistance among both mothers and their infants who become infected despite prophylaxis and the risk of increased transmission. $^{21,22}$

Infant NVP prophylaxis has the advantages of low cost while achieving comparable low transmission rates to maternal prophylaxis, lack of risk of multiclass resistance to the mother or infant and being more forgiving of imperfect adherence given the long half-life of NVP. In addition, infant NVP prophylaxis provides an effective postnatal PMTCT option for mothers who either do not tolerate or refuse maternal prophylaxis. Acknowledged disadvantages are lack of impact on horizontal transmission among discordant partners and lack of benefit for maternal health.

To optimally decrease mother-to-child transmission and reduce maternal mortality, the highest priority should be to ensure that women who currently require ART for their own health receive it. ${ }^{23}$ When there is an adequate infrastructure to determine which women require ART, the HPTN 046 find- ings support the safety and effectiveness of extended infant NVP prophylaxis in reducing the risk of transmission among asymptomatic HIV-infected breastfeeding women with higher CD4 cell counts as long it is maintained throughout the risk period of breastfeeding. In addition, given its long half-life, extended infant nevirapine remains an effective option for those HIV-infected mothers who either cannot tolerate, opt out or do not adhere to taking maternal ARVs long term.

\section{ACKNOWLEDGMENTS}

The authors gratefully acknowledge all the mothers and infants across the 4 sites who participated in the study, the staff at each of the 4 HPTN 046 sites, as well as all National Institutes of Health, the Fred Hutchinson Cancer Research Center, Family Health International, and the HPTN Central Laboratory staff who worked tirelessly to support the HPTN 046 protocol development and implementation. The authors acknowledge and thank Laura Guay (former trial co-chair) for her leadership and guidance for the protocol development and implementation, Heather Watts, NICHD, for her input into protocol development and addressing maternal safety issues, Scharla Estep, NIAID Protocol Pharmacist for her strong support to the site pharmacists and providing ongoing drug product oversight, Avinash Shetty (Wake Forest University Health Sciences) for review of safety events and input into protocol development, and Tom Fleming study team statistician from the University of Washington/Fred Hutchinson Cancer Research Center for his ongoing statistical input and unwavering support for the scientific research questions being posed in the trial.

\section{REFERENCES}

1. UNAIDS. UNAIDS Report on the Global AIDS Epidemic | 2012. Available at: http://www.unaids.org/en/media/unaids/contentassets/documents/ epidemiology/2012/gr2012/20121120_UNAIDS_Global_Report_2012_ en.pdf. Accessed September 12, 2013.

2. Bedri A, Gudetta B, Isehak A, et al. Extended-dose nevirapine to 6 weeks of age for infants to prevent HIV transmission via breastfeeding in Ethiopia, India, and Uganda: an analysis of three randomised controlled trials. Lancet. 2008;372:300-313

3. Kumwenda NI, Hoover DR, Mofenson LM, et al. Extended antiretroviral prophylaxis to reduce breast-milk HIV-1 transmission. $N$ Engl J Med. 2008;359:119-129.

4. Chasela CS, Hudgens MG, Jamieson DJ, et al. Maternal or infant antiretroviral drugs to reduce HIV-1 transmission. $N$ Engl J Med. 2010;362: 2271-2281.

5. Shapiro RL, Hughes MD, Ogwu A, et al. Antiretroviral regimens in pregnancy and breast-feeding in Botswana. N Engl J Med. 2010;362: 2282-2294

6. de Vincenzi I. Triple antiretroviral compared with zidovudine and singledose nevirapine prophylaxis during pregnancy and breastfeeding for prevention of mother-to-child transmission of HIV-1 (Kesho Bora study) a randomised controlled trial. Lancet Infect Dxis. 2011;11:171-180.

7. WHO. Guidelines on HIV and Infant Feeding 2010. PRinciples and Recommendations for Infant Feeding in the Context of HIV and a Summary of Evidence. Available at: http://www.who.int/maternal_child_ adolescent/documents/9789241599535/en/. Accessed September 12, 2013.

8. WHO. Antiretroviral Drugs for Treating Pregnant Women and Preventing HIV Infection in Infants. REcommendations for a Public Health Approach 2010 Version. Available at: http://whqlibdoc.who.int/publications/2010/ 9789241599818_eng.pdf. Accessed September 12, 2013.

9. WHO. Use of Antiretroviral Drugs for Treating Pregnant Women and Preventing HIV Infection in Infants Executive Summary April 2012. 
Available at: http://www.who.int/hiv/PMTCT_update.pdf. Accessed September 12, 2013.

10. World Health Organization HIV/AIDS Programme. Consolidated Guidelines on the Use of Antiretroviral Drug for Treatment and Preventing HIV Infection: Recommendations for a Public Health Approach. Geneva, Switzerland: 2013. Printed Kuala Lumpur Malaysia. Available at: http:// www.who.int/hiv/pub/guidelines/arv2013/intro/executivesummary/en/index. $\mathrm{html}$. Accessed September 12, 2013.

11. Coovadia HM, Brown ER, Fowler MG, et al. Efficacy and safety of an extended nevirapine regimen in infant children of breastfeeding mothers with HIV-1 infection for prevention of postnatal HIV-1 transmission (HPTN 046): a randomised, double-blind, placebo-controlled trial. Lancet. 2012;379:221-228.

12. Division of AIDS (DAIDS) Table for Grading Severity the Severity of Adult and Pediatric Adverse Events. Version 1.0; December, 2004; Clarification August 2009. Available at: http://rsc.tech-res.com/Document/ safetyandpharmacovigilance/Table_for_Grading_Severity_of_Adult_ Pediatric_Adverse_Events.pdf. Accessed September 12, 2013.

13. Taha TE, Kumwenda J, Cole SR, et al. Postnatal HIV-1 transmission after cessation of infant extended antiretroviral prophylaxis and effect of maternal highly active antiretroviral therapy. $J$ Infect Dis. 2009;200:1490-1497.

14. Jamieson DJ, Chasela CS, Hudgens MG, et al. Maternal and infant antiretroviral regimens to prevent postnatal HIV-1 transmission: 48-week follow-up of the BAN randomised controlled trial. Lancet. 2012;379:2449-2458.

15. Coutsoudis A, Dabis F, Fawzi W, et al. Late postnatal transmission of HIV-1 in breast-fed children: an individual patient data meta-analysis. $J$ Infect Dis. 2004;189:2154-2166.

16. Cohen MS, Chen YQ, McCauley M, et al. Prevention of HIV-1 infection with early antiretroviral therapy. $N$ Engl J Med. 2011;365:493-505.
17. Nachega J, Uthman O, Mills E, et al. Adherence to ART during and after Prengancy in Low, Middle, and High-Income Countries: a Systematic Review and Meta-analyses. 19th Conference on Retroviruses and Opportunistic Infections (CROI). Alexandria, VA: CROI LLC; 2012:468.

18. Clouse K, Maskew M, Bassett J, et al. Delayed Diagnosis of HIV and High Rates of Lost to Follow-up Among Pregnant Women Attending Antenatal Services at a Primary Health Clinic: Johannesburg, South Africa. 19th Conference on Retroviruses and Opportunistic Infections (CROI). Seattle, WA: Abstract 1004, Program Abstracts; 2012:467.

19. Rawizza H, Meloni S, Oyebode T, et al. Evaluation of Loss to Follow up Within the PMTCT Care Cascade in a Large ART Program: Nigeria. 19th Conference on Retroviruses and Opportunistic Infections (CROI). Alexandria, VA: CROI LLC; 2012:474.

20. Kreitchmann R, Harris R, Kakehasi F, et al. ARV Adherence during Pregnancy and Post Partum: Latin America. 19th Conference on Retroviruses and Opportunistic Infections (CROI). Alexandria, VA: CROI LLC; 2012:474.

21. Zeh C, Weidle PJ, Nafisa L, et al. HIV-1 drug resistance emergence among breastfeeding infants born to HIV-infected mothers during a single-arm trial of triple-antiretroviral prophylaxis for prevention of mother-to-child transmission: a secondary analysis. PLoS med. 2011;8:e1000430.

22. Fogel J, Li Q, Taha TE, et al. Initiation of antiretroviral treatment in women after delivery can induce multiclass drug resistance in breastfeeding HIV-infected infants. Clin Infect Dis. 2011;52:1069-1076.

23. Kuhn L, Aldrovandi GM, Sinkala M, et al. Potential impact of new WHO criteria for antiretroviral treatment for prevention of mother-to-child HIV transmission. AIDS. 2010;24:1374-1377. 